TITLE:

\title{
Bioassay Data for Marine Pollution Using Sea Urchin Eggs, 1982 and 1983
}

AUTHOR(S):

Kobayashi, Naomasa

\section{CITATION:}

Kobayashi, Naomasa. Bioassay Data for Marine Pollution Using Sea Urchin Eggs, 1982 and 1983. PUBLICATIONS OF THE SETO MARINE BIOLOGICAL LABORATORY 1985, 30(4-6): 333338

ISSUE DATE:

1985-12-25

URL:

http://hdl.handle.net/2433/176105

RIGHT: 


\section{Bioassay Data for Marine Pollution Using Sea Urchin Eggs, 1982 and 1983}

1982

Three experiments were made as follows.

I. Winter season, January 26, eggs of Hemicentrotus pulcherrimus were used, see Table 1.

II. Spring season, an experiment was made in May 24, using Anthocidaris crassispina eggs, see Table 2.

III. Autumn season, September 6, eggs of Anthocidaris crassispina were used, see Publ. Seto Mar. Biol. Lab., Vol. 30, No. 4/6, pp. 220-221, Table 5, 1985.

(Notes common to all tables: Fertilization membrane formation examined $3 \mathrm{~min}$. after fertilization; minutes and hours in parentheses respectively after First cleavage, Gastrulation and Pluteus indicate the time after insemination; the maturation state of gonads used was nearly ripe to full ripe; For the 0 min. old sperms and 3.5-6 hrs. old eggs, Degree of inhibitory effect 0 show the non-inhibtiion, 1 the slight inhibition, 2 the weak, 3 the moderate, 4 the strong and 5 the violent inhibition state of the sea water tested (see Ranking II, Publ. Seto Mar. Biol. Lab., Vol. XXI, No. 5/6, p. 391, Table 8, 1974); For the 5 mins. old sperms and 3.5-6 hrs. old eggs, Degree of inhibitory effect see the above and Ranking III, Publ. Seto Mar. Biol. Lab., Vol. 30, No. 4/6, p. 224, Table 6, 1985.

1983

Four experiments were made as follows.

I. Winter season, February 2, eggs of Hemicentrotus pulcherrimus were used, see Table 3.

II. Spring season, an experiment was made in May 13, using Anthocidaris crassispina eggs, see Table 4.

III. Summer season, July 24, eggs of Anthocidaris crassisispina were used, see Table 5. IV. Autumn season, an experiment was made in September 12, using Anthocidaris crassispina eggs, see Table 6 .

(Notes common to all tables: See the notes mentioned above.)

Naomasa Kobayashi

Biological Laboratory

Doshisha University

Kyoto, 602, Japan

Publ. Seto Mar. Biol. Lab., 30 (4/6), 333-338, 1985. (Biological Data 2) 
Table 1. Results of the Jan. 26, '82 experiment with eggs of Hemicentrotus pulcherrimus.

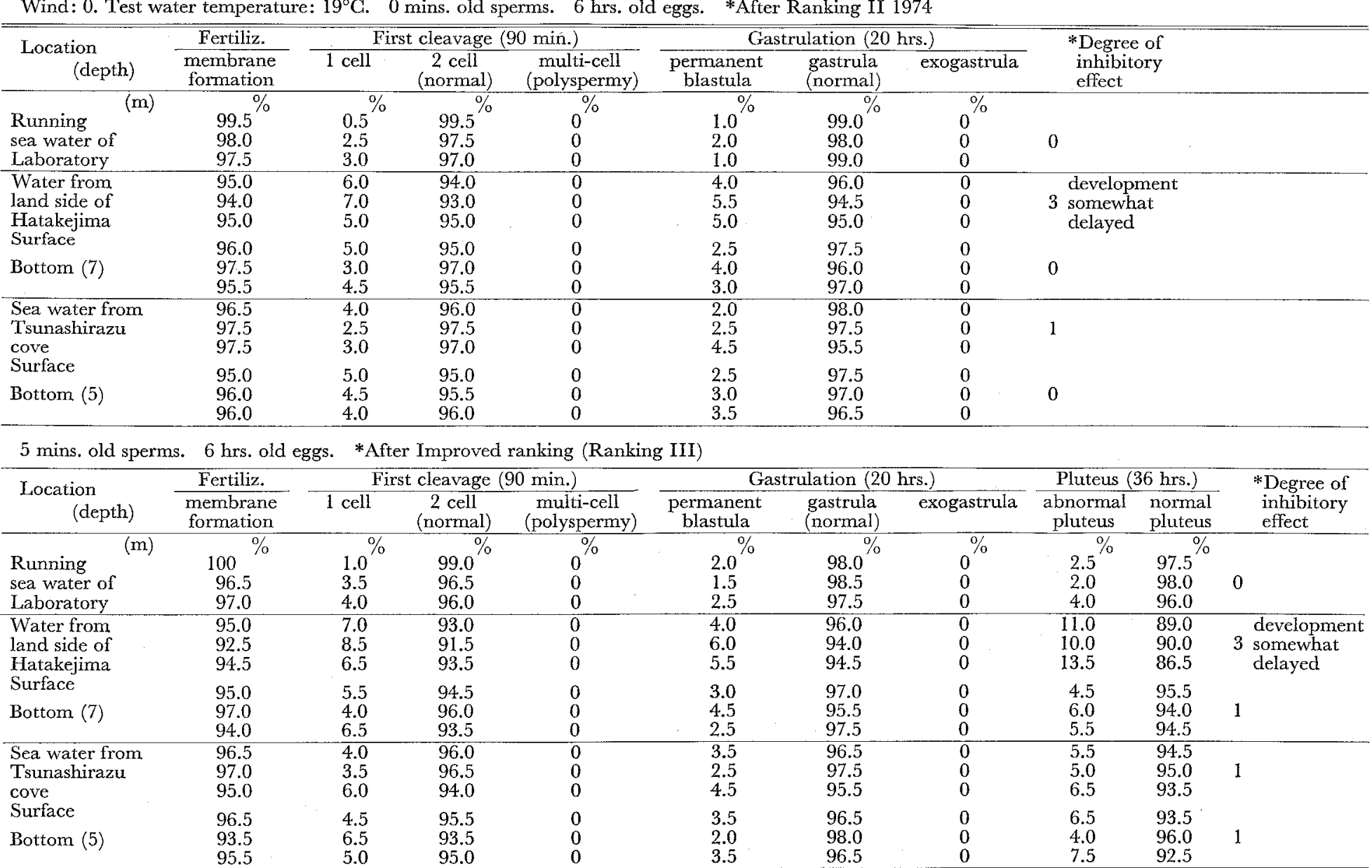


Table 2. Results of the May 24, '82 experiment with eggs of Anthocidaris crassispina.

Wind: 0 . Test water temperature: $24^{\circ} \mathrm{G}$. 0 mins. old sperms. 3.5 hrs. old eggs. *After Ranking II 1974

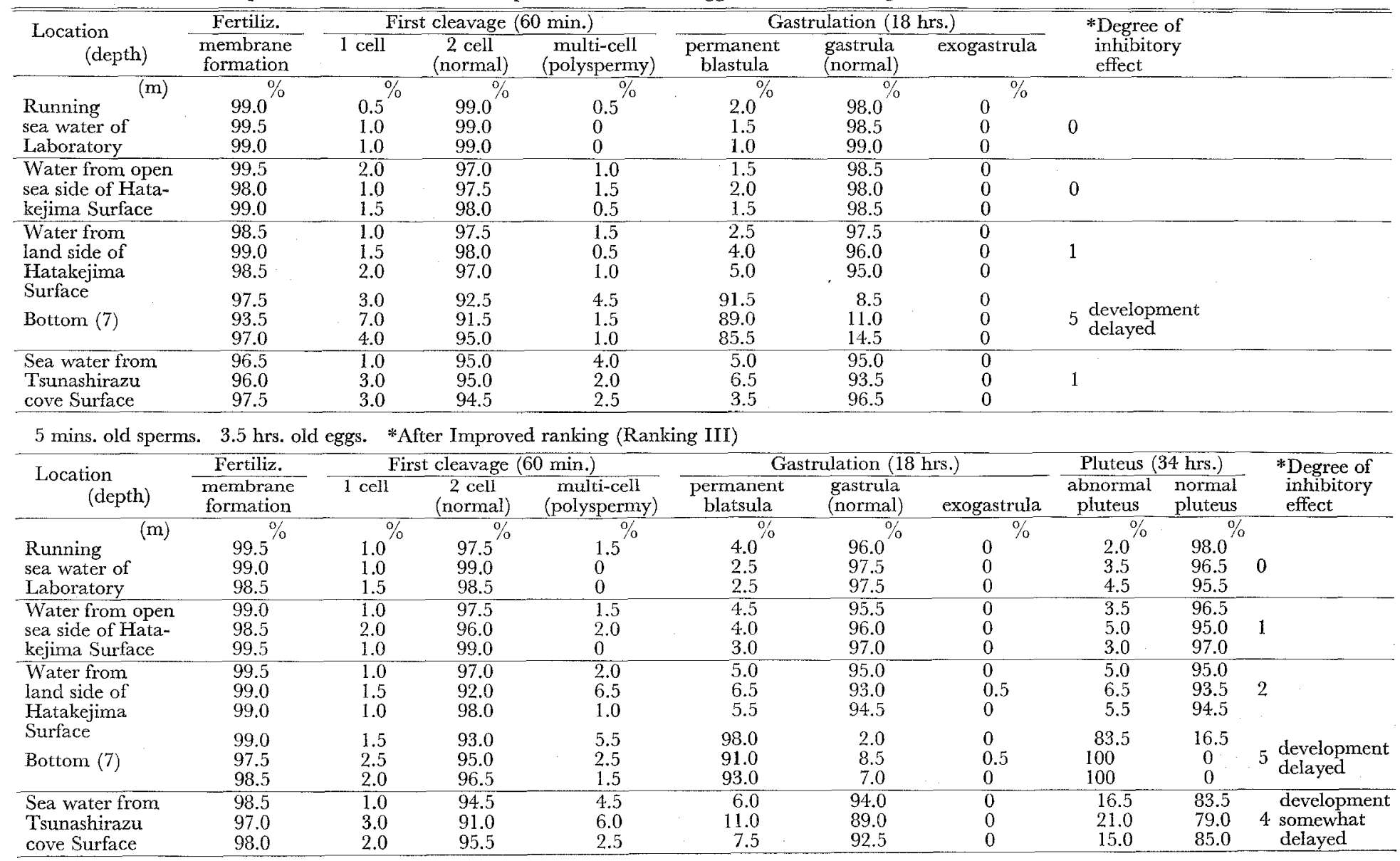


Table 3. Results of the Feb. 2, '83 experiment with eggs of Hemicentrotus pulcherrimus.

Wind: 0 . Test water temperature: $19^{\circ} \mathrm{C} .5$ mins. old sperms. 6 hrs. old eggs. *After Ranking III

\begin{tabular}{|c|c|c|c|c|c|c|c|c|c|c|}
\hline \multirow{2}{*}{$\begin{array}{l}\text { Location } \\
\qquad \text { (depth) }\end{array}$} & \multirow{2}{*}{$\begin{array}{l}\text { Fertiliz. } \\
\text { membrane } \\
\text { formation }\end{array}$} & \multicolumn{3}{|c|}{ First cleavage (90 min.) } & \multicolumn{3}{|c|}{ Gastrulation (20 hrs.) } & \multicolumn{2}{|c|}{ Pluteus (36 hrs.) } & \multirow{2}{*}{$\begin{array}{l}\text { *Degree of } \\
\text { inhibitory } \\
\text { effect }\end{array}$} \\
\hline & & $1 \mathrm{cell}$ & $\begin{array}{c}2 \text { cell } \\
\text { (normal) }\end{array}$ & $\begin{array}{c}\text { multi-cell } \\
\text { (polyspermy) }\end{array}$ & $\begin{array}{c}\text { permanent } \\
\text { blastula }\end{array}$ & $\begin{array}{l}\text { gastrula } \\
\text { (normal) }\end{array}$ & exogastrula & $\begin{array}{l}\text { abnormal } \\
\text { pluteus }\end{array}$ & $\begin{array}{l}\text { normal } \\
\text { pluteus }\end{array}$ & \\
\hline (m) & $945^{\%}$ & $60^{\%}$ & $04 \%$ & $\%$ & $\%$ & $\%$ & $\%$ & $\%$ & $\%$ & \\
\hline Running & 94.5 & 6.0 & 94.0 & 0 & 0 & 100 & 0 & $1.5^{\circ}$ & $98.5^{\prime 0}$ & \\
\hline sea water of & 95.0 & 6.5 & 93.5 & 0 & 0 & 100 & 0 & 1.0 & 99.0 & 0 \\
\hline Laboratory & 96.5 & 4.0 & 95.5 & 0.5 & 0.5 & 99.5 & 0 & 1.0 & 99.0 & \\
\hline Water from & 93.0 & 8.5 & 89.0 & 2.5 & 0.5 & 99.5 & 0 & 2.0 & 98.0 & \\
\hline land side of & 92.0 & 9.0 & 90.0 & 1.0 & 0.5 & 99.5 & 0 & 1.0 & 99.0 & 1 \\
\hline Hatakejima & 94.0 & 6.5 & 92.0 & 1.5 & 1.0 & 99.0 & 0 & 1.5 & 98.5 & \\
\hline Surface & 93.0 & 6.5 & 92.5 & 1.0 & 3.5 & 96.5 & 0 & 2.5 & 97.5 & \\
\hline Bottom (7) & 90.5 & 10.0 & 88.0 & 2.0 & 1.0 & 99.0 & 0 & 3.5 & 96.5 & 1 \\
\hline & 91.0 & 9.5 & 89.0 & 1.5 & 2.5 & 97.5 & 0 & 4.0 & 96.0 & \\
\hline Sea water from & 94.5 & 7.0 & 92.0 & 1.0 & 2.0 & 98.0 & 0 & 3.0 & 97.0 & \\
\hline Tsunashirazu & 91.5 & 8.5 & 90.5 & 1.0 & 2.0 & 98.0 & 0 & 3.0 & 97.0 & 1 \\
\hline cove & 90.0 & 11.0 & 87.0 & 2.0 & 1.5 & 98.5 & 0 & 3.0 & 97.0 & \\
\hline Surtace & 81.5 & 24.5 & 74.5 & 1.0 & 4.0 & 96.0 & 0 & 14.0 & 86.0 & \\
\hline Bottom (5) & 88.0 & 13.0 & 86.0 & 1.0 & 2.5 & 97.5 & 0 & 13.5 & 86.5 & 2 \\
\hline & 84.5 & 17.0 & 81.0 & 2.0 & 5.0 & 95.0 & 0 & 13.0 & 87.0 & \\
\hline
\end{tabular}

Table 4. Results of the May 13, '83 experiment with eggs of Anthocidaris crassispina.

Wind: 0 . Test water temperature: $25^{\circ} \mathrm{C}$. 5 mins. old sperms. $3.5 \mathrm{hrs.} \mathrm{old} \mathrm{eggs.} \mathrm{*After} \mathrm{Ranking} \mathrm{III}$

\begin{tabular}{|c|c|c|c|c|c|c|c|c|c|c|}
\hline \multirow{2}{*}{$\begin{array}{l}\text { Location } \\
\qquad \text { (depth) }\end{array}$} & \multirow{2}{*}{$\begin{array}{l}\text { Fertiliz. } \\
\text { membrane } \\
\text { formation }\end{array}$} & \multicolumn{3}{|c|}{ First cleavage $(55 \mathrm{~min})}$. & \multicolumn{3}{|c|}{ Gastrulation (17 hrs.) } & \multicolumn{2}{|c|}{ Pluteus (33 hrs.) } & \multirow{2}{*}{$\begin{array}{l}\text { * Degree of } \\
\text { inhibitory } \\
\text { effect }\end{array}$} \\
\hline & & 1 cell & $\begin{array}{c}2 \text { cell } \\
\text { (normal) }\end{array}$ & $\begin{array}{c}\text { multi-cell } \\
\text { (polyspermy) }\end{array}$ & $\begin{array}{l}\text { permanent } \\
\text { blastula }\end{array}$ & $\begin{array}{c}\text { gastrula } \\
\text { (normal) }\end{array}$ & exogastrula & $\begin{array}{l}\text { abnormal } \\
\text { pluteus }\end{array}$ & $\begin{array}{l}\text { normal } \\
\text { pluteus }\end{array}$ & \\
\hline $\begin{array}{l}\text { Running } \\
\text { sea water of } \\
\text { Laboratory }\end{array}$ & $\begin{array}{l}99.0^{\%} \\
98.0 \\
97.0\end{array}$ & $\begin{array}{l}1.5^{\%} \\
3.0 \\
3.5 \\
\end{array}$ & $\begin{array}{l}98.5 \\
97.0 \\
96.5\end{array}$ & $\begin{array}{l}0 \% \\
0 \\
0 \\
0\end{array}$ & $\begin{array}{l}2.0^{\%} \\
1.0 \\
1.5\end{array}$ & $\begin{array}{l}98.0^{\%} \\
99.0 \\
98.5\end{array}$ & $\begin{array}{l}\% \\
0 \\
0 \\
0\end{array}$ & $\begin{array}{l}0.5^{\%} \\
1.5 \\
1.0\end{array}$ & $\begin{array}{l}99.5 \\
98.5 \\
99.0\end{array}$ & 0 \\
\hline $\begin{array}{l}\text { Water from } \\
\text { open sea side } \\
\text { of Hatakejima } \\
\text { Surface }\end{array}$ & $\begin{array}{l}95.0 \\
97.5 \\
96.0\end{array}$ & $\begin{array}{l}6.0 \\
3.0 \\
5.0\end{array}$ & $\begin{array}{l}94.0 \\
97.0 \\
95.0\end{array}$ & $\begin{array}{l}0 \\
0 \\
0 \\
\end{array}$ & $\begin{array}{l}3.0 \\
2.0 \\
2.5\end{array}$ & $\begin{array}{l}97.0 \\
98.0 \\
97.5\end{array}$ & $\begin{array}{l}0 \\
0 \\
0\end{array}$ & $\begin{array}{l}1.0 \\
2.0 \\
1.5\end{array}$ & $\begin{array}{l}99.0 \\
98.0 \\
98.5\end{array}$ & 0 \\
\hline $\begin{array}{l}\text { Water from land } \\
\text { side of Hatake- } \\
\text { jima Surface }\end{array}$ & $\begin{array}{l}97.5 \\
98.0 \\
96.5\end{array}$ & $\begin{array}{l}2.5 \\
3.0 \\
3.5\end{array}$ & $\begin{array}{l}97.5 \\
97.0 \\
96.5\end{array}$ & $\begin{array}{l}0 \\
0 \\
0\end{array}$ & $\begin{array}{l}1.5 \\
2.5 \\
1.5\end{array}$ & $\begin{array}{l}98.5 \\
97.5 \\
98.5\end{array}$ & $\begin{array}{l}0 \\
0 \\
0\end{array}$ & $\begin{array}{l}0.5 \\
2.0 \\
1.0\end{array}$ & $\begin{array}{l}99.5 \\
98.0 \\
99.0\end{array}$ & 0 \\
\hline $\begin{array}{l}\text { Sea water from } \\
\text { Tsunashirazu } \\
\text { cove Surface }\end{array}$ & $\begin{array}{l}78.5 \\
83.0 \\
86.0\end{array}$ & $\begin{array}{l}23.0 \\
20.0 \\
15.5\end{array}$ & $\begin{array}{l}77.0 \\
80.0 \\
94.5\end{array}$ & $\begin{array}{l}0 \\
0 \\
0\end{array}$ & $\begin{array}{l}4.0 \\
5.5 \\
3.5\end{array}$ & $\begin{array}{l}96.0 \\
94.5 \\
96.5\end{array}$ & $\begin{array}{l}0 \\
0 \\
0\end{array}$ & $\begin{array}{l}2.0 \\
4.5 \\
3.5\end{array}$ & $\begin{array}{l}98.0 \\
95.5 \\
96.5\end{array}$ & 2 \\
\hline
\end{tabular}


Table 5. Results of the July 24, '83 experiment with eggs of Anthocidaris crassispina.

Wind: 0 . Test water temperature: $28^{\circ} \mathrm{C}$. 5 mins. old sperms. 3 hrs. old eggs. *After Ranking III

\begin{tabular}{|c|c|c|c|c|c|c|c|c|c|c|}
\hline \multirow{2}{*}{$\begin{array}{l}\text { Location } \\
\qquad \text { (depth) }\end{array}$} & \multirow{2}{*}{$\begin{array}{l}\text { Fertiliz. } \\
\begin{array}{l}\text { membrane } \\
\text { formation }\end{array}\end{array}$} & \multicolumn{3}{|c|}{ First cleavage (50 min.) } & \multicolumn{3}{|c|}{ Gastrulation (15 hrs.) } & \multicolumn{2}{|c|}{ Pluteus (26 hrs.) } & \multirow{2}{*}{$\begin{array}{l}\text { *Degree of } \\
\text { inhibitory } \\
\text { effect }\end{array}$} \\
\hline & & 1 cell & $\begin{array}{c}2 \text { cell } \\
\text { (normal) }\end{array}$ & $\begin{array}{c}\text { multi-cell } \\
\text { (polypsermy) }\end{array}$ & $\begin{array}{c}\text { permanent } \\
\text { blastula }\end{array}$ & $\begin{array}{l}\text { gastrula } \\
\text { (normal) }\end{array}$ & exogastrula & $\begin{array}{l}\text { abnormal } \\
\text { pluteus }\end{array}$ & $\begin{array}{l}\text { normal } \\
\text { pluteus }\end{array}$ & \\
\hline \multirow{3}{*}{$\begin{array}{l}\text { Running } \\
\text { sea water of } \\
\text { Laboratory }\end{array}$} & $98.0^{\%}$ & $2.5^{\%}$ & $97.5^{\%}$ & $\%$ & $1.5^{\%}$ & $98.5^{\%}$ & $0^{\%}$ & $1.0^{\%}$ & $99.0^{\%}$ & \multirow{3}{*}{0} \\
\hline & 99.0 & 2.0 & 98.0 & 0 & 1.0 & 99.0 & 0 & 1.5 & 98.5 & \\
\hline & 97.0 & 4.0 & 96.0 & 0 & 2.0 & 98.0 & 0 & 2.0 & 98.0 & \\
\hline \multirow{3}{*}{$\begin{array}{l}\text { Water from } \\
\text { open sea side } \\
\text { of Hatakejima } \\
\text { Surface }\end{array}$} & 98.5 & 3.0 & 97.0 & 0 & 1.0 & 99.0 & 0 & 0.5 & 90.5 & \multirow{3}{*}{0} \\
\hline & 99.5 & 1.5 & 98.5 & 0 & 0.5 & 99.5 & 0 & 1.0 & 99.0 & \\
\hline & 96.0 & 4.5 & 95.5 & 0 & 1.0 & 99.0 & 0 & 1.5 & 98.5 & \\
\hline \multirow{3}{*}{ Bottom (25) } & 96.0 & 5.5 & 94.5 & 0 & 1.5 & 98.5 & 0 & 2.0 & 98.0 & \multirow{3}{*}{0} \\
\hline & 94.0 & 6.0 & 93.0 & 1.0 & 2.5 & 97.5 & 0 & 1.5 & 98.5 & \\
\hline & 95.0 & 7.5 & 91.0 & 1.5 & 2.5 & 97.5 & 0 & 2.0 & 98.0 & \\
\hline \multirow{3}{*}{$\begin{array}{l}\text { Water from } \\
\text { land side of } \\
\text { Hatakejima } \\
\text { Surface }\end{array}$} & 92.0 & 9.0 & 91.0 & 0 & 2.0 & 98.0 & 0 & 3.0 & 97.0 & \multirow{3}{*}{1} \\
\hline & 93.5 & 7.0 & 92.5 & 0.5 & 2.5 & 97.5 & 0 & 4.5 & 95.5 & \\
\hline & 89.0 & 12.0 & 87.5 & 0.5 & 3.0 & 97.0 & 0 & 6.0 & 94.0 & \\
\hline \multirow{3}{*}{ Bottom (27) } & 89.0 & 12.0 & 86.0 & 2.0 & 2.0 & 98.0 & 0 & 4.5 & 95.5 & \multirow{3}{*}{2} \\
\hline & 86.0 & 11.0 & 85.5 & 3.5 & 1.5 & 98.5 & 0 & 7.0 & 93.0 & \\
\hline & 87.0 & 15.0 & 80.0 & 5.0 & 2.0 & 98.0 & 0 & 6.5 & 93.5 & \\
\hline \multirow{3}{*}{$\begin{array}{l}\text { Sea water from } \\
\text { Tsunashirazu } \\
\text { cove } \\
\text { Surface }\end{array}$} & 91.0 & 10.0 & 89.0 & 1.0 & 3.0 & 97.0 & 0 & 6.0 & 94.0 & \multirow{3}{*}{1} \\
\hline & 93.5 & 7.5 & 92.0 & 0.5 & 2.5 & 97.5 & 0 & 5.5 & 94.5 & \\
\hline & 88.0 & 12.0 & 87.0 & 1.0 & 3.0 & 97.0 & 0 & 7.5 & 92.5 & \\
\hline \multirow{3}{*}{ Bottom (5) } & 90.5 & 10.0 & 87.0 & 3.0 & 2.5 & 97.5 & 0 & 7.0 & 93.0 & \multirow{3}{*}{2} \\
\hline & 87.0 & 13.5 & 82.0 & 4.5 & 2.0 & 98.0 & 0 & 6.5 & 93.5 & \\
\hline & 88.5 & 16.0 & 82.0 & 2.0 & 3.0 & 97.0 & 0 & 8.0 & 92.0 & \\
\hline
\end{tabular}


Table 6. Results of the Sept. 12, '83 experiment with eggs of Anthocidaris crassispina.

Wind: SW1. Test water temperature: $27^{\circ} \mathrm{C} . \quad 5$ mins. old sperms. 3 hrs. old eggs. *After Ranking III

\begin{tabular}{|c|c|c|c|c|c|c|c|c|c|c|}
\hline \multirow{2}{*}{$\begin{array}{l}\text { Location } \\
\qquad \text { (depth) }\end{array}$} & \multirow{2}{*}{$\begin{array}{l}\text { Fertiliz. } \\
\text { membrane } \\
\text { formation }\end{array}$} & \multicolumn{3}{|c|}{ First cleavage (50 min.) } & \multicolumn{3}{|c|}{ Gastrulation (15 hrs.) } & \multicolumn{2}{|c|}{ Pluteus (26 hrs.) } & \multirow{2}{*}{$\begin{array}{l}\text { *Degree of } \\
\text { inhibitory } \\
\text { effect }\end{array}$} \\
\hline & & 1 cell & $\begin{array}{c}2 \text { cell } \\
\text { (normal) }\end{array}$ & $\begin{array}{c}\text { multi-cell } \\
\text { (polyspermy) }\end{array}$ & $\begin{array}{c}\text { premanent } \\
\text { blastula }\end{array}$ & $\begin{array}{l}\text { gastrula } \\
\text { (normal) }\end{array}$ & exogastrula & $\begin{array}{l}\text { abnormal } \\
\text { pluteus }\end{array}$ & $\begin{array}{l}\text { normal } \\
\text { pluteus }\end{array}$ & \\
\hline \multirow{3}{*}{$\begin{array}{l}\text { Running } \\
\text { sea water of } \\
\text { Laboratory }\end{array}$} & $99.5^{\%}$ & $1.0^{\%}$ & $99.0^{\%}$ & $\%$ & $0^{\%}$ & $100 \%$ & $0 \%$ & $0.5^{\%}$ & $99.5^{\%}$ & \\
\hline & 99.0 & 1.5 & 98.5 & 0 & 1.5 & 98.5 & 0 & 1.0 & 99.0 & 0 \\
\hline & 99.5 & 0.5 & 98.5 & 1.0 & 1.0 & 99.0 & 0 & 1.0 & 99.0 & \\
\hline \multirow{3}{*}{$\begin{array}{l}\text { Water from } \\
\text { open sea side } \\
\text { of Hatakejima } \\
\text { Surface }\end{array}$} & 99.0 & 1.0 & 99.0 & 0 & 0.5 & 99.5 & 0 & 1.5 & 98.5 & \\
\hline & 99.5 & 0.5 & 99.5 & 0 & 1.0 & 99.0 & 0 & 1.0 & 99.0 & 0 \\
\hline & 99.5 & 0.5 & 99.0 & 0.5 & 1.5 & 98.5 & 0 & 1.5 & 98.5 & \\
\hline \multirow{4}{*}{$\begin{array}{l}\text { Water from } \\
\text { land side of } \\
\text { Hatakejima } \\
\text { Surface }\end{array}$} & 96.5 & 1.5 & 80.0 & 18.5 & 7.5 & 92.5 & 0 & 13.5 & 86.5 & \\
\hline & 95.0 & 2.0 & 82.5 & 15.5 & 9.5 & 90.5 & 0 & 11.0 & 89.0 & 5 \\
\hline & 94.0 & 3.5 & 85.0 & 11.5 & 10.0 & 90.0 & 0 & 8.5 & 91.5 & \\
\hline & 93.0 & 27.0 & 69.5 & 3.5 & 12.5 & 87.5 & 0 & 11.5 & 88.5 & \\
\hline \multirow[t]{2}{*}{ Bottom (27) } & 94.5 & 21.5 & 77.0 & 1.5 & 9.0 & 91.0 & 0 & 2.5 & 97.5 & 2 \\
\hline & 95.0 & 18.5 & 77.0 & 4.5 & 11.5 & 88.5 & 0 & 7.0 & 93.0 & \\
\hline \multirow{3}{*}{$\begin{array}{l}\text { Sea water from } \\
\text { Tsunashirazu } \\
\text { cove } \\
\text { Surface }\end{array}$} & 97.0 & 10.5 & 83.0 & 6.5 & 9.5 & 90.5 & 0 & 5.5 & 94.5 & \\
\hline & 96.0 & 8.5 & 86.5 & 5.0 & 8.0 & 92.0 & 0 & 7.5 & 92.5 & 2 \\
\hline & 95.0 & 10.0 & 86.5 & 3.5 & 10.5 & 89.5 & 0 & 6.0 & 94.0 & \\
\hline \multirow{3}{*}{ Bottom (5) } & 94.5 & 25.5 & 71.5 & 3.0 & 13.5 & 86.5 & 0 & 4.5 & 95.5 & \\
\hline & 95.5 & 18.5 & 80.5 & 1.0 & 10.5 & 89.5 & 0 & 3.0 & 97.0 & 2 \\
\hline & 91.5 & 21.0 & 75.0 & 4.0 & 9.5 & 90.5 & 0 & 5.5 & 94.5 & \\
\hline
\end{tabular}

